\title{
Single-mode and multimode Fabry-Pérot interference in suspended graphene
}

\author{
Mika Oksanen, ${ }^{1}$ Andreas Uppstu, ${ }^{2}$ Antti Laitinen, ${ }^{1}$ Daniel J. Cox,${ }^{1}$ Monica F. Craciun, ${ }^{3}$ Saverio Russo, ${ }^{3}$ \\ Ari Harju, ${ }^{2}$ and Pertti Hakonen ${ }^{1}$ \\ ${ }^{1}$ Low Temperature Laboratory, Aalto University, P.O. Box 15100, FI-00076 Aalto, Finland \\ ${ }^{2}$ COMP Centre of Excellence and Helsinki Institute of Physics, Department of Applied Physics, Aalto University, FI-00076 Aalto, Finland \\ ${ }^{3}$ Centre for Graphene Science, School of Physics, University of Exeter, EX4 4QL Exeter, United Kingdom
}

(Received 15 August 2013; published 31 March 2014)

\begin{abstract}
We have achieved high-quality Fabry-Pérot interference in a suspended graphene device both in conductance and in shot noise. A Fourier analysis of these reveals two sets of overlapping, coexisting interference patterns, with the ratios of the resonance intervals being equal to the width to length ratio of the device. We show that these sets originate from the unique coexistence of longitudinal and transverse resonances, with the longitudinal resonances occurring due to bunching of modes with low transverse momentum. Finally, the high quality of our samples allows us to probe the interaction renormalization of the Fermi velocity as well as the coexistence of Fabry-Pérot oscillations with universal conductance fluctuations.
\end{abstract}

DOI: 10.1103/PhysRevB.89.121414

Phase coherence of charge carriers leads to electronwave interference in ballistic mesoscopic conductors [1]. In graphene, such Fabry-Pérot-like interference has been observed [2-6], but the two-dimensional nature of conduction together with substrate-induced disorder have prevented the full understanding of the complex interference patterns [7-9]. Experiments on suspended, exfoliated graphene have demonstrated mobilities exceeding $200000 \mathrm{~cm}^{2} /$ Vs [4,10], enabling experimental studies of ballistic transport in micrometer-sized graphene samples. The interference patterns in graphene are similar to those in SWCNTs but, principally, more involved due to the two-dimensional nature of graphene, which leads to increased complexity due to the presence of a large number of conduction channels. Nonuniform spatial conditions, like charge puddles [11] and flexural deformation [12], complicate the situation even further. As a consequence, no coherent picture of Fabry-Pérot interference in suspended graphene devices has emerged by now.

The theoretical conditions for Fabry-Pérot resonances have been analyzed in several recent works [7-9]. Gunlycke and White [7] showed that with nonperfect contacts, evenly spaced Fabry-Pérot-like resonances should be observable in conductance measurements, with the periodicity being determined by the length of the sample and the velocity of the charge carriers. Such longitudinal resonances originate from simultaneous participation of modes in nonequivalent channels, facilitated by transversely quantized states with low transverse momentum and small energy separation. Müller et al. [9] emphasized that Fabry-Pérot resonances should be observable at suitable gate voltage in experimental setups with metallic contacts, due to the presence of $p$ - $n$ junctions. Additionally, they theorized that in low temperature and with high-quality edges, resonances that correspond to single transverse modes should also be observable. Previously, equivalent

Published by the American Physical Society under the terms of the Creative Commons Attribution 3.0 License. Further distribution of this work must maintain attribution to the author(s) and the published article's title, journal citation, and DOI.
PACS number(s): 72.80.Vp, 07.60.Ly, 73.50.Td, 81.05.ue

single-mode resonances have been observed in single-wall carbon nanotubes (SWCNTs) [13].

In this work, we employ conductance and shot noise measurements to analyze transport resonances with characteristic features of Fabry-Pérot interference. Our results show resonances that are attributable to both transverse (single-mode) and longitudinal (multimode) interference. Moreover, the suspended region of the sample yields a Fermi velocity $v_{F}$ of the order of $2.4-2.8 \times 10^{6} \mathrm{~m} / \mathrm{s}$ at a charge density of $n \sim 1-2 \times 10^{10} \mathrm{~cm}^{-2}$. Although the exact value of $v_{F}$ may be overestimated because of anomalies like nonuniform charge distribution [14], both theoretical predictions and experimental observations have suggested that in suspended graphene, many-body interactions may renormalize $v_{F}$ to a significantly higher value than found in graphene on a substrate $[15,16]$.

Our experiments were performed at a temperature of $50 \mathrm{mK}$ using a suspended graphene sample with length $L=1.1 \mu \mathrm{m}$ and width $W=4.5 \mu \mathrm{m}$. The experimental setup is shown in Figs. 1(a) and 1(b). Before the measurements, the two-lead sample was annealed by passing a current of $1.1 \mathrm{~mA}$ through it (using a voltage of nearly $0.9 \mathrm{~V}$ over the sample). This resulted in an almost neutral, high-quality sample with the charge neutrality point located at $V_{g}^{\text {Dirac }}=-0.15 \mathrm{~V}$. The fieldeffect mobility was estimated to be $\mu>10^{5} \mathrm{~cm}^{2} / \mathrm{Vs}$ at charge densities of $n<2.5 \times 10^{10} \mathrm{~cm}^{-2}$. An estimate for the contact resistance, $R_{c} \lesssim 100 \Omega$, was obtained based on the measured Fano factor $\mathcal{F}=0.2$ at $n \sim 3-4 \times 10^{10} \mathrm{~cm}^{-2}$, by assuming that the total shot noise is induced by a tunneling contact with $\mathcal{F}=1$.

We measured both the differential conductance and the differential Fano factor. Lines connected to the graphene device (source and drain) were connected to bias-Ts separating low-frequency signals (less than $1 \mathrm{kHz}$ ) and the high-frequency noise measurement signals above $600 \mathrm{MHz}$, dc biasing via a $0.5-\mathrm{M} \Omega$ resistor was employed. The high-frequency line on the source side was terminated by a $50-\Omega$ shunt and the line on the drain side (shot noise measurement line) was connected to a low noise amplifier through a circulator [18]. Standard lock-in techniques were employed for measurements of the low-frequency conductance (around $35 \mathrm{~Hz}$ ), the same 
(a)

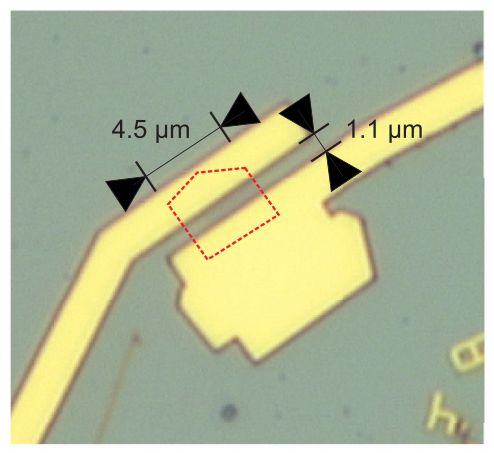

(b)

(c)
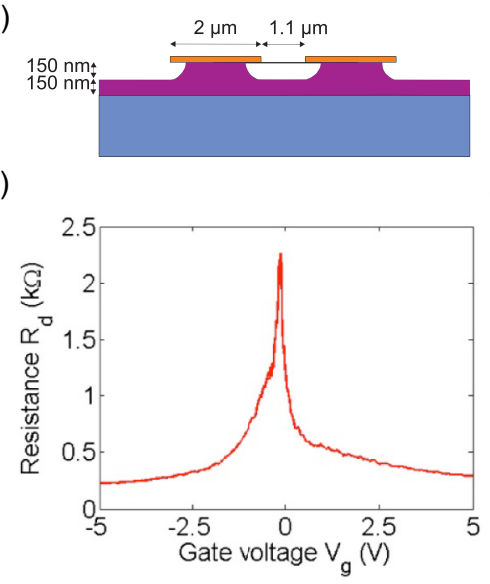

(d)

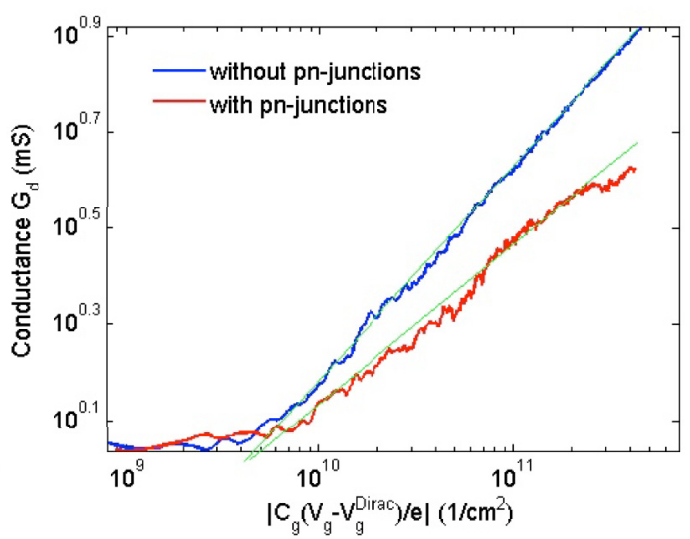

FIG. 1. (Color online) (a) Optical image of the sample, where the dashed line indicates the position of the graphene flake. (b) Schematic structure of the suspended graphene device fabricated with $\mathrm{HF}$ etching of $150 \mathrm{~nm}$ of $\mathrm{SiO}_{2}$ and following the procedure described in Ref. [17]. (c) Resistance of the sample as a function of the gate voltage up to $\pm 5 \mathrm{~V}$, which corresponds to a charge density range up to $\pm 15 \times 10^{10} \mathrm{~cm}^{-2}$. (d) Conductance vs $\left|C_{g}\left(V_{g}-V_{g}^{\text {Dirac }}\right) / e\right|$ on a $\log -\log$ scale. The solid lines yield an estimate of $n_{r} \simeq 6 \times 10^{9} \mathrm{~cm}^{-2}$ for the residual charge density.

excitation could also be employed to measure differential shot noise by directing the rectified output of the noise spectrometer to a lock-in amplifier.

As the $\mathrm{Cr} / \mathrm{Au}$ contacts of the setup cause $n$ doping of the underlying graphene, at negative gate voltage $p n$ junctions are formed close to the contacts. This causes increased reflectivity $[19,20]$, which assists in the formation of FabryPérot resonances. The measured minimum conductivity falls below $\frac{4 e^{2}}{h}$ approaching theoretical [21,22] minimum conductivity $\sigma_{\min }=\frac{4 e^{2}}{\pi h}$ for high aspect ratio samples, the actual maximum resistance of the sample was $2.2 \mathrm{k} \Omega$, as shown in Fig. 1(c). The charge density on the device at zero bias is $\left|n_{0}\right|=$ $\sqrt{C_{g}^{2}\left(V_{g}-V_{g}^{\text {Dirac }}\right)^{2} / e^{2}+n_{r}^{2}}$, where $C_{g}$ is the capacitance per unit area of the gate setup, $V_{g}^{\text {Dirac }}$ the location of the Dirac point, and $n_{r}$ the residual charge density due to impurities. $n_{r}$ can be determined using the $\ln (G)$ vs $\ln \left(\left|C_{g}\left(V_{g}-V_{g}^{\text {Dirac }}\right) / e\right|\right)$ plot shown in Fig. 1(d), based on the point where the linear behavior levels to a constant value at low $\ln \left[\left|C_{g}\left(V_{g}-V_{g}^{\text {Dirac }}\right) / e\right|\right]$. The estimation leads to a value of $n_{r} \sim 6 \times 10^{9} \mathrm{~cm}^{-2}$.

Approximate locations for Fabry-Pérot resonances in a graphene device of length $L$ and width $W$ are obtained by using the particle-in-a-box type expression [7,9]

$$
E_{q_{L}, q_{W}} \cong \pm \sqrt{E_{L}^{2}\left(q_{L}+\delta_{L}\right)^{2}+E_{W}^{2}\left(q_{W}+\delta_{W}\right)^{2}}
$$

where $E_{L} \equiv h v_{F} / 2 L$ and $E_{W} \equiv h v_{F} / 2 W$. The values of the constants $\delta_{L}$ and $\delta_{W}$ depend on the details of the interfaces or edges [7], and the numbers $q_{L}$ and $q_{W}$ label longitudinal and transverse modes, respectively. According to Eq. (1), in a device with $W / L \gg 1$, modes with low $q_{W}$ are bunched together close to the values $E_{q_{L}, 0}$, occurring with spacing $E_{L}$. This signifies a multichannel Fabry-Pérot interference phenomenon. On the other hand, at fixed $q_{L}$, modes with large $q_{W}$ are spaced roughly as $E_{W}$. The widths of the resonances are approximately given by $\delta \mu \cong \sqrt{2}\left(q_{L} \pi\right)^{2} E_{L}^{3} / \mu^{2}$
[9], with the sharpest peaks corresponding to low $q_{L}$ and high $\mu$.

We have studied the appearance of the resonances through a tight-binding transport simulation of a graphene device with dimensions $100 \times 24 \mathrm{~nm}^{2}$. Metallic contacts are modeled by semi-infinite graphene leads at a constant doping of $300 \mathrm{meV}$ (the main features of the resulting interference pattern do not depend on the exact amount of doping) and edge disorder is simulated by removing edge atoms with a probability of 0.2 and repeating the procedure five times. Figure 2(a) shows a zoom-in on the pn side of the resulting conductance curve, which clearly contains both fast transverse and slow longitudinal oscillations. The edge disorder reduces the amplitude of the transverse oscillations by a factor of three. The locations of some of the resonances given by Eq. (1) have been indicated with vertical lines. The solid lines correspond to fast transverse resonances with varying $q_{W}$ but $q_{L}$ set to one, while approximate positions for the slow longitudinal resonances are given by the dashed lines, which correspond to $q_{W}=0$ and varying $q_{L}$. The locations of the longitudinal resonances is estimated well by Eq. (1), whereas the transverse resonances are given only qualitatively due to the presence of edge disorder.

Figure 2(b) shows a simulated conductance map that highlights the presence of two sets of resonances, forming diamondlike patterns. The map is based on the Landauer approach, according to which the current flowing through the device equals at zero temperature $I=(2 e / h) \int_{\mu_{L}}^{\mu_{R}} T(E-$ $\left.E_{\text {Dirac }}\right) d E$, where $\mu_{L(R)}$ is the chemical potential of the left (right) contact, $T\left(E-E_{\text {Dirac }}\right)$ is the transmission function and $e V_{\text {bias }}=\mu_{R}-\mu_{L}$. The effect of the gate voltage is to shift the location of $E_{\text {Dirac }}$ with respect to the chemical potentials of the contacts. In the experiments, the other contact is grounded, and applying a finite bias voltage will thus also shift the location of $E_{\text {Dirac }}$ with respect to the grounded contact. Here, we assume that this shift is linear, i.e., $\Delta E_{\text {Dirac }}=x e V_{\text {bias }}$. This results in the observed diamondlike pattern of the $d I / d V_{\text {bias }}$ map, with 
(a)

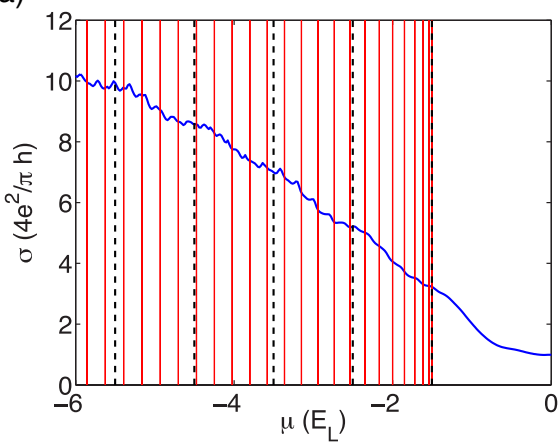

(b)

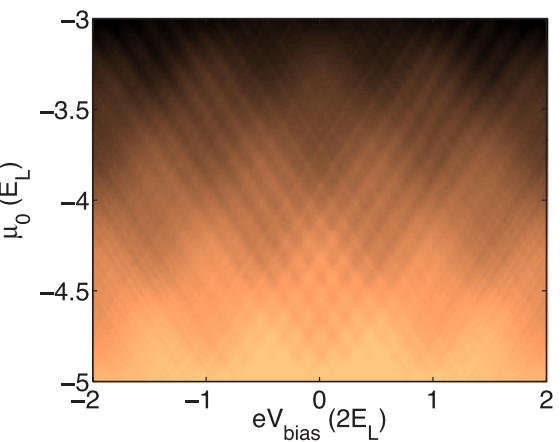

(c)

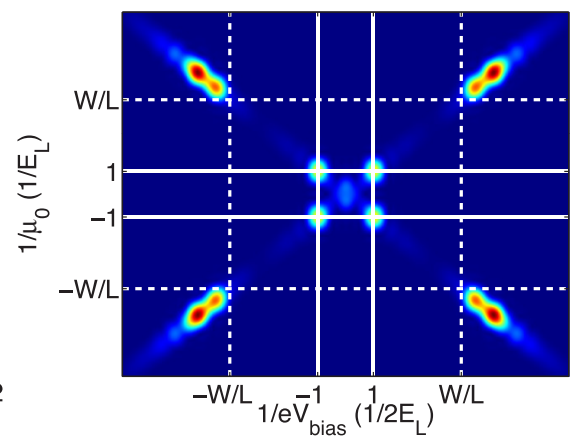

FIG. 2. (Color online) (a) Simulated conductance curve showing the presence of fast and slow oscillations, corresponding to transverse and longitudinal Fabry-Pérot interference, respectively. The lines indicate resonances given by Eq. (1), with the solid lines corresponding to $q_{L}=1$ and arbitrary $q_{W}$, and the dashed lines to $q_{W}=0$ and arbitrary $q_{L}$. (b) Simulated map of the differential conductance $G_{d}$. (c) Fourier transform of $\partial G_{d} / \partial \mu$, highlighting the periodicities of the two sets of resonances.

complete symmetry when $x=0.5$. The obtained conductance map can be used to analyze the resonances quantitatively by first differentiating it with respect to $\mu$, and then performing a Fourier transform. The Fourier transformed map is shown in Fig. 2(c), with the periodicity of $E_{L}$ being clearly visible, while the periodicity of the fast resonances being slightly lower than $E_{W}$, due to resonances corresponding to relatively low $q_{W}$ [as the red bars in Fig. 2(a) show, with growing $q_{W}$ the spacing approaches $E_{W}$ from below].
In the experiments, scans over the bias voltage $V_{\text {bias }}$ and gate voltage $V_{g}$ show a Fabry-Pérot pattern both in conductance and in shot noise. The pattern is observable on both sides of the Dirac point, but it is more clear at negative gate voltage. To quantitatively study the experimental map of the differential conductance $G_{d} \equiv d I / d V_{\text {bias }}$, shown in Fig. 3(a), we convert the gate voltage into a low-bias chemical potential $\mu_{0}$. Using the density of states of graphene, the charge density can be converted into a zero-bias chemical potential through (a)

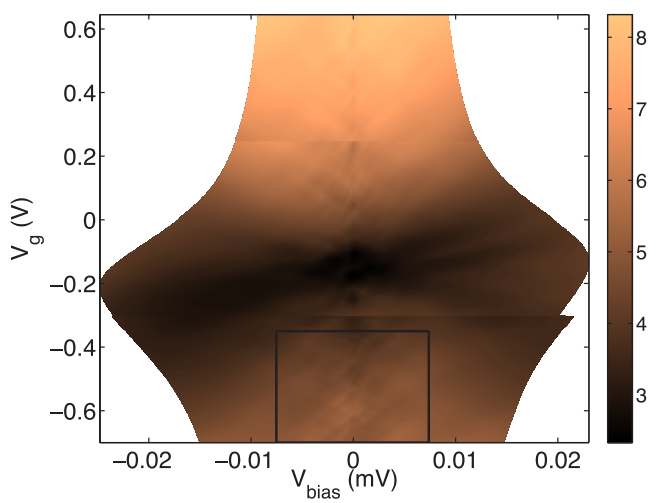

(b)



(c)

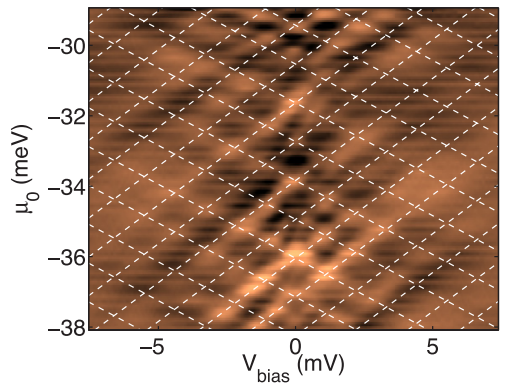

(e)

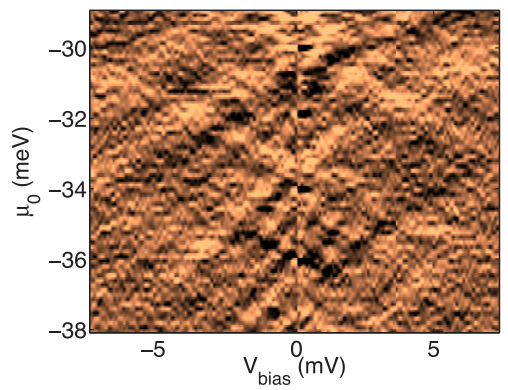

(d)

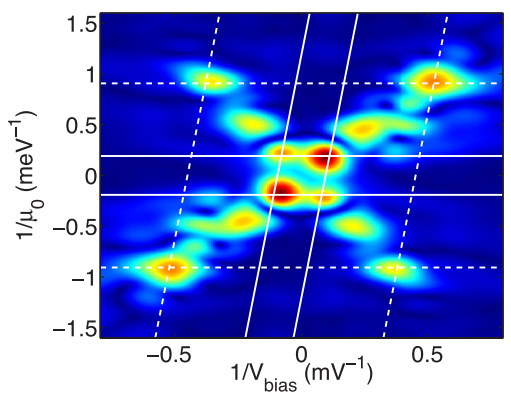

(f)

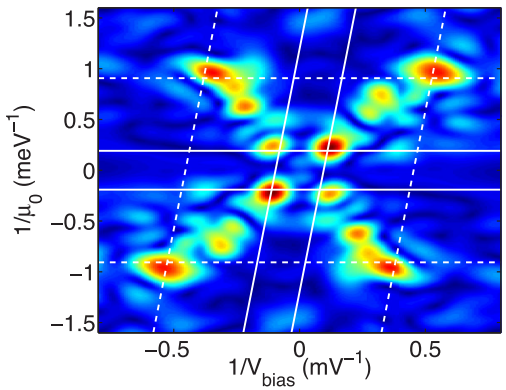

FIG. 3. (Color online) (a) Measured differential conductivity map of the graphene device. (b) A zoom-in on the boxed region in (a), with the gate voltage converted into chemical potential. (c) The conductance map in (b) differentiated with respect to the chemical potential. The dashed lines are a fit of resonances with periodicity $E_{W}$, using $v_{F}=2.4 \times 10^{6} \mathrm{~m} / \mathrm{s}$. (d) Fourier transform of (c). The solid lines are fit to the longitudinal interference and the dashed to the transverse interference, using the same $v_{F}$ as in (c). (e) Differential Fano factor differentiated with respect to the chemical potential. (f) Fourier transform of (e), with the solid and dashed lines being the same as those shown in (d). 
$\mu_{0}=\operatorname{sgn}\left(n_{0}\right) \hbar v_{F} \sqrt{\pi\left|n_{0}\right|}$. Figure 3(b) shows a zoom-in of the converted data at a charge density of $n_{0}=1.1-1.8 \times$ $10^{10} \mathrm{~cm}^{-2}$, indicated by the box in Fig. 3(a). A diamond pattern appears if the conductance is differentiated with respect to the zero-bias chemical potential. The differentiated data, displayed in Fig. 3(c), indicates that there is a periodic modulation in $G_{d}$, especially visible well away from the Dirac point, which is located around $V_{g}=-0.15 \mathrm{~V}$. A good fit with the experimental data can be obtained, if $C_{g}$ is set to the value given by a plane capacitor model of the gate setup, i.e., $47 \mathrm{aF} / \mu \mathrm{m}^{2}$, and $n_{r}$ to $9 \times 10^{9} \mathrm{~cm}^{-2}$, which is close to the value estimated using Fig. 1(d). The observed Fabry-Pérot resonances have relatively low amplitude. Weak reflection at the contact interfaces will lead to weak longitudinal interference [7], whereas edge disorder suppresses transverse resonances. In our simulation, the strength of the longitudinal resonances is determined by the amount of doping of the graphene leads.

The diamonds are not completely symmetric, which can be explained through slightly asymmetrical contacts. A best fit with the Landauer approach described earlier is achieved by setting $x=0.58$. By setting $W=4.5 \mu \mathrm{m}$ and $v_{F}=2.4 \times$ $10^{6} \mathrm{~m} / \mathrm{s}$, we obtain the dashed lines shown in Fig. 3(c). However, if we assume that the average periodicity of the fast resonances is slightly less than $E_{W}$, as in the simulation, we obtain the estimate $v_{F} \simeq 2.8 \times 10^{6} \mathrm{~m} / \mathrm{s}$. Hence our measurements yield $v_{F}=2.4-2.8 \times 10^{6} \mathrm{~m} / \mathrm{s}$ at $n \sim 1-2 \times 10^{10} \mathrm{~cm}^{-2}$. The large Fermi velocity, well above values measured on a $\mathrm{SiO}_{2}$ substrate [23,24], is consistent with measurements of the cyclotron mass in freestanding graphene [15], which have indicated that $v_{F}$ is between 2 and $3 \times 10^{6} \mathrm{~m} / \mathrm{s}$ at similar charge density. This effect is thought to be caused by unscreened electron-electron interactions occurring in suspended graphene [16]. As in the simulation, Fourier analysis may be applied to reveal the presence of multimode resonances spaced as $E_{L}$. In the Fourier-transformed plot, shown in Fig. 3(d), a strong resonance is indeed found at a periodicity that corresponds to a value close to $L=1.1 \mu \mathrm{m}$. The fast, transverse resonances, indicated by the dashed lines in Fig. 3(d), correspond to dashed diamond pattern in Fig. 3(c).

Shot noise yields complementary information on the distribution of transmission channels as well as interaction effects $[25,26]$. Shot noise can be quantified through the differential Fano factor, which is defined as $\mathcal{F}_{d} \equiv(1 / 2 e) d S / d I$, where $S$ is the correlation function of the current fluctuations $\delta I(t)$, i.e., $S=\int d t\langle\delta I(t) \delta I(0)+\delta I(0) \delta I(t)\rangle$. Our measured results on shot noise are illustrated in Fig. 3(e), which depicts the derivative $\partial \mathcal{F}_{d} / \partial \mu$ as a function of $\mu_{0}$ and $V_{\text {bias }}$ over the same area as in Fig. 3(b). Although the resonances in Fig. 3(e) are not straightforward to interpret, the Fourier transform shown in Fig. 3(f) reveals two clear sets of resonances with the same periodicities as the conductance data, with the solid and dashed lines corresponding to exactly the same values as in Fig. 3(d). The Fourier transforms of both the $\partial G_{d} / \partial \mu$ and $\partial \mathcal{F}_{d} / \partial \mu$ maps contain additional spots between the innermost and outermost resonances, which indicate the presence of additional scattering.

A detailed analysis of $G_{d}\left(V_{\text {bias }}, \mu\right)$ and $F_{d}\left(V_{\text {bias }}, \mu\right)$ indicates the coexistence of periodic universal conductance fluctuations (UCF) and Fabry-Pérot oscillations at chemical potentials of 30-40 meV [26-39]. This conclusion is reached by making a careful comparison of the experimental results against tight binding simulations at a realistic density of resonant scatterers; the nature and distribution of the scatterers were selected to agree with the measured variance of zero-bias conductance fluctuations versus gate voltage near the Dirac point. The analysis indicates that the UCF oscillations can lead to periodic modulation on top of the Fabry-Pérot pattern and thereby produce additional peak structures in the Fourier spectra, both in conductance and noise analysis. This additional structure is identified with the intermediate peaks in the Fourier spectra of Fig. 3. The positions of these peaks were influenced by a small external field, which renders further support to the conclusion of the impurity-induced peak patterns in the Fourier spectra.

To summarize, we have measured the conductance of a suspended graphene sample with a high aspect ratio. Through Fourier analysis techniques, the differential conductance and the differential shot noise reveal two sets of clear resonances, which may be attributed to single-mode transverse and multimode longitudinal resonance. The analysis of the interference pattern yields a Fermi velocity that is significantly higher than the one usually reported for graphene on a substrate, thus indicating that close to the Dirac point, unscreened manybody interactions become significant in suspended graphene. Furthermore, we find that universal conductance fluctuations, the dominant mechanism for $\delta G$ around the Dirac point, may coexist with Fabry-Pérot oscillations at $|\mu|=30-40 \mathrm{meV}$ and their (quasi)periodicity yields specific signatures in the Fourier spectra.

This work has been supported in part by the EU 7th Framework Programme (Grant Nos. 228464 Microkelvin and EU-FP7-NMP-246026), by the Academy of Finland (project Nos. 251748, 250280 LTQ CoE, and 135908), and by the European Science Foundation (ESF) under the EUROCORES Programme EuroGRAPHENE. SR and MFC acknowledge financial support from EPSRC (EP/J000396/1, EP/K017160/1, and $\mathrm{EP} / \mathrm{K} 010050 / 1)$
[1] S. Datta, Electronic Transport in Mesoscopic Systems (Cambridge University Press, Cambridge, 1997).

[2] F. Miao, S. Wijeratne, Y. Zhang, U. C. Coskun, W. Bao, and C. N. Lau, Science 317, 1530 (2007).

[3] H. B. Heersche, P. Jarillo-Herrero, J. B. Oostinga, L. M. K. Vandersypen, and A. F. Morpurgo, Nature 446, 56 (2007).
[4] X. Du, I. Skachko, A. Barker, and E. Y. Andrei, Nat. Nanotech. 3, 491 (2008).

[5] A. F. Young and P. Kim, Nat. Phys. 5, 222 (2009).

[6] P. Rickhaus, R. Maurand, M.-H. Liu, M. Weiss, K. Richter, and C. Schönenberger, Nat. Commun. 4, 2342 (2013).

[7] D. Gunlycke and C. T. White, Appl. Phys. Lett. 93, 122106 (2008). 
[8] A. V. Shytov, M. S. Rudner, and L. S. Levitov, Phys. Rev. Lett. 101, 156804 (2008).

[9] M. Müller, M. Bräuninger, and B. Trauzettel, Phys. Rev. Lett. 103, 196801 (2009).

[10] K. I. Bolotin, K. J. Sikes, J. Hone, H. L. Stormer, and P. Kim, Phys. Rev. Lett. 101, 096802 (2008).

[11] Y. Zhang, V. W. Brar, C. Girit, A. Zettl, and M. F. Crommie, Nat. Phys. 5, 722 (2009).

[12] E. V. Castro, H. Ochoa, M. I. Katsnelson, R. V. Gorbachev, D. C. Elias, K. S. Novoselov, A. K. Geim, and F. Guinea, Phys. Rev. Lett. 105, 266601 (2010).

[13] W. Liang, M. Bockrath, D. Bozovic, J. H. Hafner, M. Tinkham, and H. Park, Nature (London) 411, 665 (2001).

[14] A. L. Grushina, D.-K. Ki, and A. F. Morpurgo, Appl. Phys. Lett. 102, 223102 (2013).

[15] D. C. Elias, R. V. Gorbachev, A. S. Mayorov, S. V. Morozov, A. A. Zhukov, P. Blake, L. A. Ponomarenko, I. V. Grigorieva, K. S. Novoselov, F. Guinea, and A. K. Geim, Nat. Phys. 7, 701 (2011).

[16] V. N. Kotov, B. Uchoa, V. M. Pereira, F. Guinea, and A. H. Castro Neto, Rev. Mod. Phys. 84, 1067 (2012).

[17] T. Khodkov, F. Withers, D. C. Hudson, M. F. Craciun, and S. Russo, Appl. Phys. Lett. 100, 013114 (2012).

[18] R. Danneau, F. Wu, M. F. Craciun, S. Russo, M. Y. Tomi, J. Salmilehto, A. F. Morpurgo, and P. J. Hakonen, J. Low Temp. Phys. 153, 374 (2008).

[19] V. V. Cheianov and V. I. Fal'ko, Phys. Rev. B 74, 041403 (2006).

[20] V. V. Cheianov, V. Fal'ko, and B. L. Altshuler, Science 315, 1252 (2007).

[21] M. I. Katsnelson, Eur. Phys. J. B 51, 157 (2006).

[22] J. Tworzydło, B. Trauzettel, M. Titov, A. Rycerz, and C. W. J. Beenakker, Phys. Rev. Lett. 96, 246802 (2006).

[23] K. S. Novoselov, A. K. Geim, S. V. Morozov, D. Jiang, M. I. Katsnelson, I. V. Grigorieva, S. V. Dubonos, and A. A. Firsov, Nature (London) 438, 197 (2005).

[24] Y.Zhang, Y.-W. Tan, H. L. Stormer, and P. Kim, Nature (London) 438, 201 (2005).
[25] R. Danneau, F. Wu, M. F. Craciun, S. Russo, M. Y. Tomi, J. Salmilehto, A. F. Morpurgo, and P. J. Hakonen, Phys. Rev. Lett. 100, 196802 (2008).

[26] F. Wu, P. Queipo, A. Nasibulin, T. Tsuneta, T. H. Wang, E. Kauppinen, and P. J. Hakonen, Phys. Rev. Lett. 99, 156803 (2007).

[27] See Supplemental Material at http://link.aps.org/supplemental/ 10.1103/PhysRevB.89.121414 for a detailed analysis of the coexistence of periodic universal conductance fluctuations and Fabry-Pérot oscillations.

[28] D. K. Ferry and S. M. Goodnick, Transport in Nanostructures (Cambridge University Press, Cambridge, 1997).

[29] M. Y. Kharitonov and K. B. Efetov, Phys. Rev. B 78, 033404 (2008).

[30] J. Berezovsky, M. F. Borunda, E. J. Heller, and R. M. Westervelt, Nanotechnol. 21, 274013 (2010).

[31] D. W. Horsell, A. K. Savchenko, F. V. Tikhonenko, K. Kechedzhi, I. V. Lerner, and V. I. Fal'ko, Solid State Commun. 149, 1041 (2009).

[32] T. O. Wehling, S. Yuan, A. I. Lichtenstein, A. K. Geim, and M. I. Katsnelson, Phys. Rev. Lett. 105, 056802 (2010).

[33] A. Ferreira, J. Viana-Gomes, J. Nilsson, E. R. Mucciolo, N. M. R. Peres, and A. H. Castro Neto, Phys. Rev. B 83, 165402 (2011).

[34] Z. H. Ni, L. A. Ponomarenko, R. R. Nair, R. Yang, S. Anissimova, I. V. Grigorieva, F. Schedin, P. Blake, Z. X. Shen, E. H. Hill, K. S. Novoselov, and A. K. Geim, Nano Lett. 10, 3868 (2010).

[35] J.-H. Chen, W. G. Cullen, C. Jang, M. S. Fuhrer, and E. D. Williams, Phys. Rev. Lett. 102, 236805 (2009).

[36] J. P. Robinson, H. Schomerus, L. Oroszlány, and V. I. Fal'ko, Phys. Rev. Lett. 101, 196803 (2008).

[37] A. Rycerz, J. Tworzydlo, and C. W. J. Beenakker, Europhys. Lett. 79, 57003 (2007).

[38] E. Rossi, J. H. Bardarson, M. S. Fuhrer, and S. Das Sarma, Phys. Rev. Lett. 109, 096801 (2012).

[39] C. Ojeda-Aristizabal, M. Monteverde, R. Weil, M. Ferrier, S. Gueron, and H. Bouchiat, Phys. Rev. Lett. 104, 186802 (2010). 